\title{
Normality of meromorphic functions and differential polynomials share values
}

\author{
Wenjun Yuan ${ }^{1,2}$, Jinchun Lai ${ }^{1 *}$, Zifeng Huang ${ }^{1}$ and Zhi Liu'
}

\section{"Correspondence:}

190534202@qq.com

'school of Mathematics and

Information Science, Guangzhou

University, Guangzhou, 510006,

China

Full list of author information is

available at the end of the article

\begin{abstract}
In this paper, we discuss the normality of meromorphic functions which involves differential polynomial sharing values. We obtain two results: Let $k$ be a positive integer, $b(\neq 0)$ be a complex number, and $h(z)$ be a polynomial with degree at least 2 , and $H\left(f, f^{\prime}, \ldots, f^{(k)}\right)$ be a differential polynomial with $\left.\frac{\Gamma}{\gamma}\right|_{H}<k+1$. Let $\mathcal{F}$ be a family of meromorphic functions defined in $D$, all of whose zeros have multiplicity at least $k+1$. If $h(z)-1$ has at least two distinct zeros, $h\left(f^{(k)}\right)+H\left(f, f^{\prime}, \ldots, f^{(k)}\right)-1$ has at most one distinct zero in $D$ for each $f \in \mathcal{F}$, then $\mathcal{F}$ is normal in $D$. If $h(z)-b$ has at least two distinct zeros and for each pair of functions $f$ and $g$ in $\mathcal{F}, h\left(f^{(k)}\right)+H\left(f, f^{\prime}, \ldots, f^{(k)}\right)$ and $h\left(g^{(k)}\right)+H\left(g, g^{\prime}, \ldots, g^{(k)}\right)$ share $b$ in $D$, then $\mathcal{F}$ is normal in $D$, too. Two examples show that a condition in our results is necessary and our results improve Fang and Hong's, and Zeng's corresponding results.
\end{abstract}

MSC: Primary 30D35; secondary 34A05

Keywords: differential polynomial; meromorphic functions; shared values; normal families

\section{Introduction and main results}

Let $D$ be a domain in $\mathbb{C}$, and $\mathcal{F}$ be a family of meromorphic functions defined in the domain $D . \mathcal{F}$ is said to be normal in $D$, in the sense of Montel, if for every sequence $\left\{f_{n}\right\} \subseteq \mathcal{F}$ contains a subsequence $\left\{f_{n_{j}}\right\}$ such that $f_{n_{j}}$ converges spherically uniformly on compact subsets of $D$.

$\mathcal{F}$ is said to be normal at a point $z_{0} \in D$ if there exists a neighborhood of $z_{0}$ in which $\mathcal{F}$ is normal. It is well known that $\mathcal{F}$ is normal in a domain $D$ if and only if it is normal at each of its points.

Let $f$ and $g$ be meromorphic functions defined in a domain $D$, and $a$ and $b$ be complex numbers. If $g(z)=b$ whenever $f(z)=a$, we write $f(z)=a \Rightarrow g(z)=b$. If $f(z)=a \Rightarrow g(z)=b$ and $g(z)=b \Rightarrow f(z)=a$, we write $f(z)=a \Longleftrightarrow g(z)=b$; If $f(z)=a \Longleftrightarrow g(z)=a$, we say that $f$ and $g$ share the value $a$ in $D$.

Let $n_{0}, n_{1}, \ldots, n_{k}$ be non-negative integers and one of them nonzero at least, and set

$$
\begin{aligned}
& M\left(f, f^{\prime}, \ldots, f^{(k)}\right)=f^{n_{0}}\left(f^{\prime}\right)^{n_{1}} \cdots\left(f^{(k)}\right)^{n_{k}}, \\
& \gamma_{M}=n_{0}+n_{1}+n_{2}+\cdots+n_{k}, \\
& \Gamma_{M}=n_{0}+2 n_{1}+3 n_{2}+\cdots+(k+1) n_{k} .
\end{aligned}
$$

@2014 Yuan et al.; licensee Springer. This is an Open Access article distributed under the terms of the Creative Commons Attribution License (http://creativecommons.org/licenses/by/2.0), which permits unrestricted use, distribution, and reproduction in any medium, provided the original work is properly cited. 
$M\left(f, f^{\prime}, \ldots, f^{(k)}\right)$ is called the differential monomial of $f, \gamma_{M}$ the degree of $M\left(f, f^{\prime}, \ldots, f^{(k)}\right)$ and $\Gamma_{M}$ the weight of $M\left(f, f^{\prime}, \ldots, f^{(k)}\right)$.

Let $M_{1}\left(f, f^{\prime}, \ldots, f^{(k)}\right), M_{2}\left(f, f^{\prime}, \ldots, f^{(k)}\right), \ldots, M_{m}\left(f, f^{\prime}, \ldots, f^{(k)}\right)$ be differential monomials of $f$, and let $a_{1}(z), a_{2}(z), \ldots, a_{m}(z)$ be analytic in $D$. Set

$$
\begin{aligned}
& H\left(f, f^{\prime}, \ldots, f^{(k)}\right)=a_{1}(z) M_{1}\left(f, f^{\prime}, \ldots, f^{(k)}\right)+\cdots+a_{m}(z) M_{m}\left(f, f^{\prime}, \ldots, f^{(k)}\right), \\
& \gamma_{H}=\max \left\{\gamma_{M_{1}}, \gamma_{M_{2}}, \ldots, \gamma_{M_{m}}\right\}, \\
& \Gamma_{H}=\max \left\{\Gamma_{M_{1}}, \Gamma_{M_{2}}, \ldots, \Gamma_{M_{m}}\right\} .
\end{aligned}
$$

$H\left(f, f^{\prime}, \ldots, f^{(k)}\right)$ is called a differential polynomial of $f, \gamma_{H}$ the degree of $H\left(f, f^{\prime}, \ldots, f^{(k)}\right)$ and $\Gamma_{H}$ the weight of $H\left(f, f^{\prime}, \ldots, f^{(k)}\right)$. If $\gamma_{M_{1}}=\gamma_{M_{2}}=\cdots=\gamma_{M_{m}}=t$, then $H\left(f, f^{\prime}, \ldots, f^{(k)}\right)$ is called a homogeneous differential polynomial of degree $t$. Set

$$
\left.\frac{\Gamma}{\gamma}\right|_{H}=\max \left\{\frac{\Gamma_{M_{1}}}{\gamma_{M_{1}}}, \frac{\Gamma_{M_{2}}}{\gamma_{M_{2}}}, \ldots, \frac{\Gamma_{M_{m}}}{\gamma_{M_{m}}}\right\} .
$$

The following theorem was proved by Fang and Hong [1].

Theorem 1.1 [1] Let $\mathcal{F}$ be a family of meromorphic functions defined in $D, k$ and $q(\geq 2)$ be two positive integers, and $H\left(f, f^{\prime}, \ldots, f^{(k)}\right)$ be a differential polynomial with $\left.\frac{\Gamma}{\gamma}\right|_{H}<k+1$. If the zeros of $f(z)$ are of multiplicity at least $k+1$ and $\left(f^{(k)}\right)^{q}+H\left(f, f^{\prime}, \ldots, f^{(k)}\right) \neq 1$ for each $f \in \mathcal{F}$, then $\mathcal{F}$ is normal in $D$.

It is natural to ask whether the condition in Theorem 1.1 that $\left(f^{(k)}\right)^{q}+H\left(f, f^{\prime}, \ldots, f^{(k)}\right) \neq 1$ can be relaxed. In this paper we investigate this problem and prove the following result.

Theorem 1.2 Let $\mathcal{F}$ be a family of meromorphic functions defined in $D, k$ be a positive integer, let $h(z)$ be a polynomial with degree at least 2 , and $H\left(f, f^{\prime}, \ldots, f^{(k)}\right)$ be a differential polynomial with $\left.\frac{\Gamma}{\gamma}\right|_{H}<k+1$. If $h(z)-1$ has at least two distinct zeros, the zeros off $(z)$ are of multiplicity at least $k+1$ and $h\left(f^{(k)}\right)+H\left(f, f^{\prime}, \ldots, f^{(k)}\right)-1$ has at most one distinct zero in $D$ for each $f \in \mathcal{F}$, then $\mathcal{F}$ is normal in $D$.

By the idea of shared values, very recently, Zeng [2] proved the following theorem.

Theorem 1.3 [2] Let $k$ and $q(\geq 2)$ be two positive integers, $b \neq 0$ be a complex number, and let $H\left(f, f^{\prime}, \ldots, f^{(k)}\right)$ be a differential polynomial with $\left.\frac{\Gamma}{\gamma}\right|_{H}<k+1$. Let $\mathcal{F}$ be a family of meromorphic functions defined in $D$, all of whose zeros have multiplicity at least $k+1$. If for each pair of functions $f$ and $g$ in $\mathcal{F},\left(f^{(k)}\right)^{q}+H\left(f, f^{\prime}, \ldots, f^{(k)}\right)$ and $\left(g^{(k)}\right)^{q}+H\left(g, g^{\prime}, \ldots, g^{(k)}\right)$ share $b$ in $D$, then $\mathcal{F}$ is normal in $D$.

It is natural to ask whether Theorem 1.3 can be improved. In this paper, we study this problem and obtain the following theorem.

Theorem 1.4 Let $k$ be a positive integer, $b(\neq 0)$ be a complex number, $h(z)$ be a polynomial, and let $H\left(f, f^{\prime}, \ldots, f^{(k)}\right)$ be a differential polynomial with $\left.\frac{\Gamma}{\gamma}\right|_{H}<k+1$. Let $\mathcal{F}$ be a family of meromorphic functions defined in $D$, all of whose zeros have multiplicity at least $k+1$. If $h(z)-b$ has at least two distinct zeros and for each pair of functions $f$ and $g$ in $\mathcal{F}, h\left(f^{(k)}\right)+$ $H\left(f, f^{\prime}, \ldots, f^{(k)}\right)$ and $h\left(g^{(k)}\right)+H\left(g, g^{\prime}, \ldots, g^{(k)}\right)$ share $b$ in $D$, then $\mathcal{F}$ is normal in $D$. 
Example 1.1 Let $D=\{z:|z|<1\}, h(z)=z^{k+1}+1$ and $\mathcal{F}:=\left\{f_{n}(z)=n z^{k+1}\right\}$. Then

$$
h\left(f_{n}^{(k)}(z)\right)+f_{n}(z)=n\left[n^{k}((k+1) !)^{k+1}+1\right] z^{k+1}+1 .
$$

We can see that $\left.\frac{\Gamma}{\gamma}\right|_{H}=1<k+1, h\left(f_{n}^{(k)}(z)\right)+f_{n}(z)-1$ has only one distinct zero in $D$ for each function $f_{n}$ in $\mathcal{F}$, and $h\left(f_{n}^{(k)}(z)\right)+f_{n}(z)$ and $h\left(f_{m}^{(k)}(z)\right)+f_{m}(z)$ share 1 in $D$ for each pair of functions $f_{n}$ and $f_{m}$ in $\mathcal{F}$. On the other hand, $f_{j}(0)=0, f_{j}\left(\frac{1}{j \frac{1}{k+1}}\right)=1$, for any $j \in \mathbb{N}$. This implies that the family $\mathcal{F}$ fails to be equicontinuous at 0 , and thus $\mathcal{F}$ is not normal at 0 .

Remark 1.5 This example shows that $h(z)-1$ to have at least two distinct zeros $(h(z)-b$ to have at least two distinct zeros) is necessary in Theorem 1.2 (Theorem 1.4).

Example 1.2 Let $D=\{z:|z|<1\}, h(z)=z^{k+1}+z^{k}+1$ and $\mathcal{F}:=\left\{f_{n}(z)=z^{k+1}\right\}$. Then

$$
h\left(f_{n}^{(k)}(z)\right)-[(k+1) !]^{k+1} f_{n}(z)+f_{n}^{\prime}(z)=\left[((k+1) !)^{k}+k+1\right] z^{k}+1
$$

We can see that $\left.\frac{\Gamma}{\gamma}\right|_{H}=2<k+1$ if $k \geq 2$, and for each pair of functions $f_{n}$ and $f_{m}$ in $\mathcal{F}$, $h\left(f_{n}^{(k)}(z)\right)-[(k+1) !]^{k+1} f_{n}(z)+f_{n}^{\prime}(z)$ and $h\left(f_{m}^{(k)}(z)\right)-[(k+1) !]^{k+1} f_{m}(z)+f_{m}^{\prime}(z)$ share 1 . Therefore, $\mathcal{F}$ is normal in $D$ by our Theorem 1.4 .

Remark 1.6 From this example we also know that $h\left(f_{n}^{(k)}(z)\right)-[(k+1) !]^{k+1} f_{n}(z)+f_{n}^{\prime}(z)-1$ has only one solution in $D$ for each $f_{n}$ in $\mathcal{F}$. The case of shared $b$ includes the case of $\neq b$, that is to say, Theorem 1.2 is a generalization of Theorem 1.1 and Theorem 1.4 is a generalization of Theorem 1.3.

\section{Preliminary lemmas}

In order to prove our results, we need the following lemmas. The first one is Zalcman's Theorem.

Lemma 2.1 [3] Let $k \in \mathbf{N}_{+}$, let $\mathcal{F}$ be a family of functions meromorphic on the unit disc $\Delta$, all of whose zeros have multiplicity at least $k$, and suppose that there exists $A \geq 1$ such that $\left|f^{(k)}(z)\right| \leq A$ whenever $f(z)=0$. Then if $\mathcal{F}$ is not normal at $z_{0}$, there exist, for each $0 \leq \alpha \leq k$,

(a) functions $f_{n} \in \mathcal{F}$;

(b) points $z_{n} \in \Delta, z_{n} \rightarrow z_{0}$, and

(c) positive numbers $\rho_{n} \rightarrow 0^{+}$

such that $g_{n}(\zeta)=\rho_{n}^{-\alpha} f_{n}\left(z_{n}+\rho_{n} \zeta\right) \rightarrow g(\zeta)$ locally uniformly with respect to the spherical metric, where $g$ is a nonconstant meromorphic function on $\mathbb{C}$, all of whose zeros have multiplicity at least $k$, such that $g^{\#}(\zeta) \leq g^{\#}(0)=k A+1$. In particular, $g$ has order at most 2 .

Here $g^{\#}(z)$ denotes the spherical derivative

$$
g^{\#}(z)=\frac{\left|g^{\prime}(z)\right|}{1+|g(z)|^{2}} .
$$

Lemma 2.2 [4] Let $f(z)=a_{n} z^{n}+a_{n-1} z^{n-1}+\cdots+a_{0}+q(z) / p(z)$, where $a_{0}, a_{1}, \ldots, a_{n}$ are constants with $a_{n} \neq 0$, and $q$ and $p$ are two co-prime polynomials, neither of which vanishes 
identically, with $\operatorname{deg} q<\operatorname{deg} p$, and let $k$ be a positive integer and $b$ a nonzero complex number. If $f^{(k)} \neq b$, and the zeros of $f$ all have multiplicity at least $k+1$, then

$$
f(z)=\frac{b(z-d)^{k+1}}{k !(z-c)}
$$

where $c$ and $d$ are distinct complex numbers.

Lemma 2.3 Let $g$ be a nonconstant meromorphic function, and $h(z)$ be a polynomial. If $h(z)$ has at least two distinct zeros and all zeros of $g$ have multiplicity at least $k+1$, then $h\left(g^{(k)}(\xi)\right)$ has at least two distinct zeros.

Proof Case 1. If $h\left(g^{(k)}(\xi)\right)$ has only one zero $\alpha$, then $h\left(g^{(k)}(\alpha)\right)=0$, and $\xi \neq \alpha, h\left(g^{(k)}(\xi)\right) \neq 0$.

Suppose that $d_{i}(i=1,2)$ are two distinct zeros of $h(z)$. Without loss of generality, we may assume that $g^{(k)}(\alpha)=d_{1}$, then $g^{(k)}(\xi) \neq d_{1}$ for $\xi \neq \alpha$.

Firstly, we will show that $g(\xi)$ is not a transcendental meromorphic function. By Nevanlinna Theory, we have

$$
\begin{aligned}
T\left(r, g^{(k)}\right) & \leq \bar{N}\left(r, g^{(k)}\right)+\bar{N}\left(r, \frac{1}{g^{(k)}-d_{1}}\right)+\bar{N}\left(r, \frac{1}{g^{(k)}-d_{2}}\right)+S\left(r, g^{(k)}\right) \\
& \leq \frac{1}{k+1} N\left(r, g^{(k)}\right)+O(\log r)+S\left(r, g^{(k)}\right) \\
& \leq \frac{1}{k+1} T\left(r, g^{(k)}\right)+O(\log r)+S\left(r, g^{(k)}\right) .
\end{aligned}
$$

Hence, we get $T\left(r, g^{(k)}\right)=O(\log r)+S\left(r, g^{(k)}\right)$, it follows that $g(\xi)$ is not a transcendental meromorphic function.

If $g(\xi)$ is a polynomial, then

$$
\begin{aligned}
h\left(g^{(k)}(\xi)\right) & =h\left[d_{1}+c(\xi-\alpha)^{n}\right] \\
& =(\xi-\alpha)^{n} Q(\xi),
\end{aligned}
$$

where $Q$ is a polynomial such that $Q(\alpha) \neq 0$ and the degree of $Q$ is not less than 1 . Thus there exists an $\alpha_{1} \neq \alpha$, such that $Q\left(\alpha_{1}\right)=0$. That is to say, there exists an $\alpha_{1} \neq \alpha$, such that $h\left(g^{(k)}\left(\alpha_{1}\right)\right)=0$, which is a contradiction.

Therefore $g(\xi)$ is rational but not a polynomial. Under the conditions of Lemma 2.2 on the rational functions $g$, we have

$$
g(\xi)=\frac{d_{2}(\xi-d)^{k+1}}{k !(\xi-c)}
$$

where $c$ and $d$ are distinct complex numbers, $d_{2} \neq 0$, and then

$$
g^{(k)}=d_{2}+\frac{A}{(\xi-c)^{k+1}}
$$

where $A \neq 0$ is a complex number.

Hence $g^{(k)}(\xi)=d_{1}$ has $k+1$ distinct zeros, which contradicts $g^{(k)}(\xi)=d_{1}$ having only the zero $\xi=\alpha$. 
Case 2. $h\left(g^{(k)}(\xi)\right) \neq 0$. Since $\operatorname{deg} h \geq 2$, by Nevanlinna Theory once more, we have

$$
\begin{aligned}
T\left(r, g^{(k)}\right) & \leq \bar{N}\left(r, g^{(k)}\right)+\bar{N}\left(r, \frac{1}{g^{(k)}-d_{1}}\right)+\bar{N}\left(r, \frac{1}{g^{(k)}-d_{2}}\right)+S\left(r, g^{(k)}\right) \\
& \leq \frac{1}{k+1} N\left(r, g^{(k)}\right)+S\left(r, g^{(k)}\right) \\
& \leq \frac{1}{k+1} T\left(r, g^{(k)}\right)+S\left(r, g^{(k)}\right),
\end{aligned}
$$

where $d_{i}(i=1,2)$ are two distinct zeros of $h(z)$. Hence, we get $T\left(r, g^{(k)}\right)=S\left(r, g^{(k)}\right)$, and it follows that $g^{(k)}$ is a constant. This together with the fact that the zeros of $g$ have multiplicity at least $k+1$ shows that $g$ is a constant, a contradiction.

\section{Proofs of theorems}

Proof of Theorem 1.2 We show that $\mathcal{F}$ is normal in $D$. Otherwise, there exists at least one point $z_{0} \in D$ such that $\mathcal{F}$ is not normal at $z_{0}$. Then by Lemma 2.1, we can find a subsequence of $\mathcal{F}$, which we may denote by $\left\{f_{n}\right\}, z_{n} \in \Delta, z_{n} \rightarrow z_{0}$ and $\rho_{n} \rightarrow 0^{+}$such that $g_{n}(\xi)=\rho_{n}^{-k} f_{n}\left(z_{n}+\rho_{n} \xi\right)$ converges local uniformly with respect to the spherical metric to a nonconstant meromorphic function $g$ on $\mathbb{C}$, all of whose zeros have multiplicity at least $k+1$.

It is easily seen that

$$
\begin{aligned}
& H\left(f_{n}, f_{n}^{\prime}, \ldots, f_{n}^{(k)}\right)\left(z_{n}+\rho_{n} \xi\right) \\
& \quad=\sum_{i=1}^{m} a_{i}\left(z_{n}+\rho_{n} \xi\right) \rho_{n}^{(k+1) \gamma_{M_{i}}-\Gamma_{M_{i}}} M_{i}\left(g_{n}, g_{n}^{\prime}, \ldots, g_{n}^{(k)}\right)(\xi) .
\end{aligned}
$$

Noting that all $a_{i}(z)(i=1,2, \ldots, m)$ are analytic on $D$ implies

$$
\left|a_{i}\left(z_{n}+\rho_{n} \xi\right)\right| \leq M\left(\frac{1+r}{2}, a_{i}(z)\right)<\infty
$$

for sufficiently large $n$, we deduce from $\left.\frac{\Gamma}{\gamma}\right|_{H}<k+1$ that

$$
\sum_{i=1}^{m} a_{i}\left(z_{n}+\rho_{n} \xi\right) \rho_{n}^{(k+1) \gamma_{M_{i}}-\Gamma_{M_{i}}} M_{i}\left(g_{n}, g_{n}^{\prime}, \ldots, g_{n}^{(k)}\right)(\xi)
$$

converges uniformly to 0 on $\mathbb{C}$.

Thus we find that

$$
h\left(g_{n}^{(k)}(\xi)\right)+\sum_{i=1}^{m} a_{i}\left(z_{n}+\rho_{n} \xi\right) \rho_{n}^{(k+1) \gamma_{M_{i}}-\Gamma_{M_{i}}} M_{i}\left(g_{n}, g_{n}^{\prime}, \ldots, g_{n}^{(k)}\right)(\xi)-1
$$

converges local uniformly to $h\left(g^{(k)}(\xi)\right)-1$ on $\mathbb{C}$.

Hence, by Hurwitz's Theorem, the hypothesis of the theorem, and Lemma 2.3, we see that $h\left(g^{(k)}(\xi)\right) \equiv 1$ or $h\left(g^{(k)}(\xi)\right)-1$ has at least two distinct zeros on $\mathbb{C}$.

Case 1. If $h\left(g^{(k)}(\xi)\right) \equiv 1$ on $\mathbb{C}$. 
Then by $h(z)-1$ having at least two distinct zeros, we find that $h(z)-c$ has at least two distinct zeros except for at most one complex number $c$. Therefore Lemma 2.3 tells us that $h\left(g^{(k)}(\xi)\right)-c$ has zero for at least two distinct $c$ except that $g(z)$ is a constant function. This is also impossible.

Case 2. If $h\left(g^{(k)}(\xi)\right)-1$ has at least two distinct zeros on $\mathbb{C}$.

Then, without out loss generality, let $\xi_{0}$ and $\xi_{0}^{\star}$ be two distinct zeros of $h\left(g^{(k)}(\xi)\right)-1$, and choose $\delta(>0)$ small enough such that $D\left(\xi_{0}, \delta\right) \cap D\left(\xi_{0}^{\star}, \delta\right)=\emptyset$, where $D\left(\xi_{0}, \delta\right)=\left\{\xi:\left|\xi-\xi_{0}\right|<\right.$ $\delta\}$, and $D\left(\xi_{0}^{\star}, \delta\right)=\left\{\xi:\left|\xi-\xi_{0}^{\star}\right|<\delta\right\}$. By Hurwitz's Theorem, there exist two sequences of points $\xi_{n} \rightarrow \xi_{0}$ and $\xi_{n}^{\star} \rightarrow \xi_{0}^{\star}$ such that for sufficiently large $n$

$$
\begin{aligned}
& h\left(f_{n}^{(k)}\right)\left(z_{n}+\rho_{n} \xi_{n}\right)+H\left(f_{n}, f_{n}^{\prime}, \ldots, f_{n}^{(k)}\right)\left(z_{n}+\rho_{n} \xi_{n}\right)-1=0, \\
& h\left(f_{n}^{(k)}\right)\left(z_{n}+\rho_{n} \xi_{n}^{\star}\right)+H\left(f_{n}, f_{n}^{\prime}, \ldots, f_{n}^{(k)}\right)\left(z_{n}+\rho_{n} \xi_{n}^{\star}\right)-1=0 .
\end{aligned}
$$

Hence, we have $\xi_{n} \in D\left(\xi_{0}, \delta\right)$ and $\xi_{n}^{\star} \in D\left(\xi_{0}^{\star}, \delta\right)$ for sufficiently large $n$. Thus each $h\left(f_{n}^{(k)}\right)(z)+H\left(f_{n}, f_{n}^{\prime}, \ldots, f_{n}^{(k)}\right)(z)-1$ has two distinct zeros for large enough $n$, which contradicts our hypothesis.

This contradiction shows that $\mathcal{F}$ is normal in $D$ and hence Theorem 1.2 is proved.

Proof of Theorem 1.4 Suppose that $\mathcal{F}$ is a family meromorphic and not normal in $D$. Then there exists at least one point $z_{0} \in D$ such that $\mathcal{F}$ is not normal at the point $z_{0}$. By Lemma 2.1, there exist:

(a) functions $f_{n} \in \mathcal{F}$;

(b) points $z_{n} \in \Delta, z_{n} \rightarrow z_{0}$, and

(c) positive numbers $\rho_{n} \rightarrow 0^{+}$

such that $g_{n}(\zeta)=\rho_{n}^{-k} f_{n}\left(z_{n}+\rho_{n} \zeta\right) \rightarrow g(\zeta)$ locally uniformly with respect to the spherical metric, where $g$ is a nonconstant meromorphic function on $\mathbb{C}$, all of whose zeros have multiplicity at least $k+1$.

It is easily seen that

$$
\begin{aligned}
& H\left(f_{n}, f_{n}^{\prime}, \ldots, f_{n}^{(k)}\right)\left(z_{n}+\rho_{n} \xi\right) \\
& \quad=\sum_{i=1}^{m} a_{i}\left(z_{n}+\rho_{n} \xi\right) \rho_{n}^{(k+1) \gamma_{M_{i}}-\Gamma_{M_{i}}} M_{i}\left(g_{n}, g_{n}^{\prime}, \ldots, g_{n}^{(k)}\right)(\xi) .
\end{aligned}
$$

Noting that all $a_{i}(z)(i=1,2, \ldots, m)$ are analytic on $D$ implies

$$
\left|a_{i}\left(z_{n}+\rho_{n} \xi\right)\right| \leq M\left(\frac{1+r}{2}, a_{i}(z)\right)<\infty
$$

for sufficiently large $n$, we deduce from $\left.\frac{\Gamma}{\gamma}\right|_{H}<k+1$ that

$$
\sum_{i=1}^{m} a_{i}\left(z_{n}+\rho_{n} \xi\right) \rho_{n}^{(k+1) \gamma_{M_{i}}-\Gamma_{M_{i}}} M_{i}\left(g_{n}, g_{n}^{\prime}, \ldots, g_{n}^{(k)}\right)(\xi)
$$

converges uniformly to 0 on $\mathbb{C}$. 
Thus we know that

$$
h\left(g_{n}^{(k)}(\xi)\right)+\sum_{i=1}^{m} a_{i}\left(z_{n}+\rho_{n} \xi\right) \rho_{n}^{(k+1) \gamma_{M_{i}}-\Gamma_{M_{i}}} M_{i}\left(g_{n}, g_{n}^{\prime}, \ldots, g_{n}^{(k)}\right)(\xi)-b
$$

converges local uniformly to $h\left(g^{(k)}(\xi)\right)-b$ on $\mathbb{C}$.

Take $f \in\left\{f_{n}\right\}$, we consider two cases.

Case 1. $\left[h\left(f^{(k)}\right)+H\left(f, f^{\prime}, \ldots, f^{(k)}\right)\right]\left(z_{0}\right) \neq b$.

Then there exists a positive number $\delta>0$ such that

$$
\left[h\left(f_{n}^{(k)}\right)+H\left(f_{n}, f_{n}^{\prime}, \ldots, f_{n}^{(k)}\right)\right](z) \neq b
$$

for all $z$ in $D_{\delta}=\left\{z:\left|z-z_{0}\right|<\delta\right\}$, by sharing condition.

Hence, by Hurwitz's Theorem, the hypothesis of the theorem, and Lemma 2.3, we see that $h\left(g^{(k)}(\xi)\right) \neq b$ or $h\left(g^{(k)}(\xi)\right) \equiv b$ on $\mathbb{C}$.

If $h\left(g^{(k)}(\xi)\right) \neq b$, then by Lemma 2.3 and the hypothesis of the theorem, we see that $h\left(g^{(k)}(\xi)\right)-b$ has at least two distinct zeros except that $g(z)$ is a constant function, a contradiction.

If $h\left(g^{(k)}(\xi)\right) \equiv b$, the same arguments of the proof of Case 1 in the proof of Theorem 1.3 implies that it does not hold.

Case 2. $\left[h\left(f^{(k)}\right)+H\left(f, f^{\prime}, \ldots, f^{(k)}\right)\right]\left(z_{0}\right)=b$.

Next we consider two subcases.

Subcase 2.1. $\left[h\left(f^{(k)}\right)+H\left(f, f^{\prime}, \ldots, f^{(k)}\right)\right](z) \equiv b$ for all $z$ in $D_{\delta}=\left\{z:\left|z-z_{0}\right|<\delta\right\}$. From the discussion above, we have $h\left(g^{(k)}(\xi)\right) \equiv b$ in $\mathbb{C}$. This is impossible.

Subcase 2.2. There exists a $\delta>0$ such that $h\left(f^{(k)}\right)+H\left(f, f^{\prime}, \ldots, f^{(k)}\right) \neq b$ in $D_{\delta}^{0}=\{z: 0<$ $\left|z-z_{0}\right|<\delta$. By the supposition and discussion above, this means that

$$
\left[h\left(f_{n}^{(k)}\right)+H\left(f_{n}, f_{n}^{\prime}, \ldots, f_{n}^{(k)}\right)\right]\left(z_{n}+\rho_{n} \xi\right) \neq b
$$

for $z_{n}+\rho_{n} \xi \neq z_{0}$, and

$$
\left[h\left(f^{(k)}\right)+H\left(f, f^{\prime}, \ldots, f^{(k)}\right)\right]\left(z_{0}\right)=b .
$$

We claim that $h\left(g^{(k)}(\xi)\right)-b$ has just a unique zero.

Suppose that there exist two distinct zeros $\xi_{0}$ and $\xi_{0}^{\star}$, choose $\delta(>0)$ small enough such that $D\left(\xi_{0}, \delta\right) \cap D\left(\xi_{0}^{\star}, \delta\right)=\emptyset$, where $D\left(\xi_{0}, \delta\right)=\left\{\xi:\left|\xi-\xi_{0}\right|<\delta\right\}$ and $D\left(\xi_{0}^{\star}, \delta\right)=\left\{\xi:\left|\xi-\xi_{0}^{\star}\right|<\delta\right\}$.

By Hurwitz's Theorem, there exist points $\xi_{n} \in D\left(\xi_{0}, \delta\right), \xi_{n}^{\star} \in D\left(\xi_{0}^{\star}, \delta\right)$, such that for sufficiently large $j$

$$
\begin{aligned}
& h\left(f_{n}^{(k)}\right)\left(z_{n}+\rho_{n} \xi_{n}\right)+H\left(f_{n}, f_{n}^{\prime}, \ldots, f_{n}^{(k)}\right)\left(z_{n}+\rho_{n} \xi_{n}\right)-b=0, \\
& h\left(f_{n}^{(k)}\right)\left(z_{n}+\rho_{n} \xi_{n}^{\star}\right)+H\left(f_{n}, f_{n}^{\prime}, \ldots, f_{n}^{(k)}\right)\left(z_{n}+\rho_{n} \xi_{n}^{\star}\right)-b=0 .
\end{aligned}
$$

By the assumption that $h\left(f^{(k)}\right)+H\left(f, f^{\prime}, \ldots, f^{(k)}\right)$ and $h\left(g^{(k)}\right)+H\left(g, g^{\prime}, \ldots, g^{(k)}\right)$ share $b$ in $D$ for each pair of functions $f$ and $g$ in $\mathcal{F}$, we see that for any integer $m$

$$
\begin{aligned}
& h\left(f_{m}^{(k)}\right)\left(z_{n}+\rho_{n} \xi_{n}\right)+H\left(f_{m}, f_{m}^{\prime}, \ldots, f_{m}^{(k)}\right)\left(z_{n}+\rho_{n} \xi_{n}\right)-b=0, \\
& h\left(f_{m}^{(k)}\right)\left(z_{n}+\rho_{n} \xi_{n}^{\star}\right)+H\left(f_{m}, f_{m}^{\prime}, \ldots, f_{m}^{(k)}\right)\left(z_{n}+\rho_{n} \xi_{n}^{\star}\right)-b=0 .
\end{aligned}
$$


We fix $m$ and note that $z_{n}+\rho_{n} \xi_{n} \rightarrow z_{0}, z_{n}+\rho_{n} \xi_{n}^{\star} \rightarrow z_{0}$ if $n \rightarrow \infty$. From this we deduce

$$
h\left(f_{m}^{(k)}\right)\left(z_{0}\right)+H\left(f_{m}, f_{m}^{\prime}, \ldots, f_{m}^{(k)}\right)\left(z_{0}\right)-b=0 .
$$

Since

$$
\left[h\left(f_{n}^{(k)}\right)+H\left(f_{n}, f_{n}^{\prime}, \ldots, f_{n}^{(k)}\right)\right]\left(z_{n}+\rho_{n} \xi\right) \neq b
$$

if $z_{n}+\rho_{n} \xi \neq z_{0}$, and

$$
\left[h\left(f^{(k)}\right)+H\left(f, f^{\prime}, \ldots, f^{(k)}\right)\right]\left(z_{0}\right)=b,
$$

noting that the zeros of

$$
h\left(f_{m}^{(k)}\right)(z)+H\left(f_{m}, f_{m}^{\prime}, \ldots, f_{m}^{(k)}\right)(z)-b
$$

have no accumulation point, for sufficiently large $n$, we have

$$
z_{n}+\rho_{n} \xi_{n}=z_{0}, \quad z_{n}+\rho_{n} \xi_{n}^{\star}=z_{0} .
$$

Hence

$$
\xi_{n}=\frac{z_{0}-z_{n}}{\rho_{n}}, \quad \xi_{n}^{\star}=\frac{z_{0}-z_{n}}{\rho_{n}} .
$$

This contradicts the fact that $\xi_{n} \in D\left(\xi_{0}, \delta\right), \xi_{n}^{\star} \in D\left(\xi_{0}^{\star}, \delta\right)$, and $D\left(\xi_{0}, \delta\right) \cap D\left(\xi_{0}^{\star}, \delta\right)=\emptyset$. So $h\left(g^{(k)}(\xi)\right)-b$ has just a unique zero ignoring multiplicity. This contradicts the conclusion of Lemma 2.3 that $h\left(g^{(k)}(\xi)\right)-b$ has at least two distinct zeros.

Hence $\mathcal{F}$ is normal at $z_{0}$, and then $\mathcal{F}$ is normal in $D$. The proof of Theorem 1.4 is complete.

\section{Competing interests}

The authors declare that they have no competing interests.

\section{Authors' contributions}

$J \mathrm{~L}$ and $\mathrm{ZH}$ carried out the design of the study and performed the analysis. WY and ZL participated in its design and coordination. All authors read and approved the final manuscript.

\section{Author details}

${ }^{1}$ School of Mathematics and Information Science, Guangzhou University, Guangzhou, 510006, China. ${ }^{2}$ Key Laboratory of Mathematics and Interdisciplinary Sciences of Guangdong Higher Education Institutes, Guangzhou University, Guangzhou, 510006, China.

\section{Acknowledgements}

This work was supported by the Visiting Academic Sponsor Project of Department of Mathematics and Statistics at Curtin University of Technology (200001807894), the first author would like to express his hearty thanks to Curtin University of Technology for providing him with very comfortable research environment. This work was completed with the support with the NSF of China (11271090) and NSF of Guangdong Province (S2012010010121). The authors wish also specially to thank the managing editor and referees for their very helpful comments and useful suggestions. 
References

1. Fang, ML, Hong, W: Some results on normal family of meromorphic functions. Bull. Malays. Math. Soc. 23, 143-151 (2000)

2. Zeng, CP: Normality and shared values. Indian J. Math. Reprint

3. Pang, XC, Zalcman, L: Normal families and shared values. Bull. Lond. Math. Soc. 32, 325-331 (2000)

4. Wang, YF, Fang, ML: Picard values and normal families of meromorphic functions with zeros. Acta Math. Sin. New Ser. 14, 17-26 (1998)

10.1186/1687-1847-2014-120

Cite this article as: Yuan et al.: Normality of meromorphic functions and differential polynomials share values. Advances in Difference Equations 2014, 2014:120

Submit your manuscript to a SpringerOpen ${ }^{\circ}$ journal and benefit from:

- Convenient online submission

- Rigorous peer review

- Immediate publication on acceptance

- Open access: articles freely available online

- High visibility within the field

- Retaining the copyright to your article

Submit your next manuscript at $>$ springeropen.com 